\title{
Importância do gerenciamento local para uma atenção primária à saúde nos moldes de Alma-Ata
}

\author{
Luceime Olivia Nunes, ${ }^{1}$ Elen Rose Lodeiro Castanheira, ${ }^{1}$ Adriano Dias, ${ }^{1}$ \\ Thais Fernanda Tortorelli Zarili, ${ }^{1}$ Patrícia Rodrigues Sanine, ${ }^{1}$ Carolina Siqueira \\ Mendonça, ${ }^{1}$ José Fernando Casquel Monti, ${ }^{2}$ Josiane Fernandes Lozigia Carrapato, ${ }^{1}$ \\ Nádia Placideli ${ }^{1}$ e Maria Ines Battistella Nemes ${ }^{3}$
}

Como citar Nunes LO, Castanheira ERL, Dias A, Zarili TFT, Sanine RR, Mendonça CS, et al. Importância do gerenciamento local para uma atenção primária à saúde nos moldes de Alma-Ata. Rev Panam Salud Publica. 2018;42:e 175. https:/ / doi.org/10.26633/RPSP.2018.175

RESUMO

Objetivo. Descrever as características da gerência das unidades de atenção primária à saúde e o perfil dos gerentes e discutir as implicações desses elementos para a efetivação dos pressupostos do Sistema Único de Saúde no Brasil de forma coerente com as proposições de Alma-Ata.

Métodos. Estudo descritivo, transversal, com dados colhidos pelo questionário de Avaliação da Qualidade de Serviços de Atenção Básica (QualiAB), um instrumento autoaplicado via web. O QualiAB foi respondido voluntariamente por 157 gerentes de unidades básicas de saúde de 41 municípios do estado de São Paulo entre outubro e dezembro de 2014.

Resultados. Das 157 unidades, 67 (42,7\%) eram unidades de saúde da família e 58 (36,9\%) eram unidades básicas de saúde de organização "tradicional"; 95 (60,5\%) se localizavam em região urbana periférica. No momento do estudo, oito (5,0\%) unidades não possuíam gerente e oito $(5,0 \%)$ eram gerenciadas por secretários municipais de saúde. Quase $80 \%$ dos gerentes eram enfermeiros e desempenhavam múltiplas funções além da gerência. O matriciamento (supervisão técnica como forma de educação permanente) era feito em 75 (47,7\%) unidades; 60 $(38,2 \%)$ unidades não contavam com nenhum tipo de matriciamento. A participação em processos avaliativos foi referida por $130(82,8 \%)$ serviços. Os principais desdobramentos induzidos por avaliações foram planejamento e reprogramação das atividades com participação da equipe multiprofissional em 40 unidades $(25,5 \%$ ) e definição de um plano anual de atividades em 38 (24,2\%). Não tiveram acesso aos resultados das avaliações 29 unidades (17,8\%).

Conclusão. O estudo recoloca a importância da gestão do trabalho e a necessidade de (re) investir na formação e valorização do gerenciamento local como estratégia para efetivar uma atenção primária à saúde capaz de promover a saúde como direito e condição de cidadania.

Palavras-chave Gestão em saúde; administração de serviços de saúde; atenção primária à saúde; Brasil.

\footnotetext{
Universidade Estadual Paulista Júlio de Mesquita Filho, Faculdade de Medicina de Botucatu, Botucatu (SP) Brasil. Correspondência: Luceime
} Olivia Nunes, olivia@fmb.unesp.br

\footnotetext{
Universidade de São Paulo (USP), Faculdade de Medicina, São Paulo (SP), Brasil.
}

Universidade Federal de São Carlos (UFSCar), Centro de Ciências Biológicas e da Saúde, São Carlos (SP), Brasil. 
A Conferência Internacional sobre Atenção Primária à Saúde (APS) realizada em Alma-Ata, em 1978, definiu a APS como chave para a implementação de um sistema de saúde que promova o desenvolvimento social e a saúde como direito (1). As recomendações de Alma-Ata, ao lado de estudos que demostram maior efetividade e eficiência de sistemas baseados numa APS forte (2), influenciaram o sistema de saúde brasileiro no sentido de priorizar um maior investimento na APS, especialmente a partir da implantação do Sistema Único de Saúde (SUS) nos anos 1990. Com o SUS, a APS, ou atenção básica, conforme denominada pela política brasileira, foi instituída como principal porta de entrada no sistema e como responsável pela ordenação do cuidado integral e articulação com as redes de atenção à saúde (RAS) (3-5). A execução das ações de APS passou a ser responsabilidade da gestão municipal, amparando-se no exercício de práticas gerenciais e sanitárias democráticas e participativas, sob forma de trabalho em equipe, conforme a Política Nacional de Atenção Básica (PNAB) (3-5).

Tomando-se por foco a dimensão organizacional, para que a APS possa cumprir o papel previsto pelo SUS faz-se necessária uma gerência das unidades básicas de saúde (UBS) capaz de coordenar a equipe de modo a orientar o processo de trabalho segundo os objetivos e finalidades propostos para esse nível de atenção. Nesse sentido, é atribuição da gerência traduzir o projeto sanitário definido pelas políticas públicas, construindo, com a equipe local e a comunidade, estratégias que transformem princípios em ações, ou seja, que traduzam fundamentos políticos em práticas concretas nos serviços de saúde (6-9).

A gerência das UBS é apontada por Weirich et al. (10) como cenário onde os problemas podem ser identificados, atendidos ou encaminhados para outros níveis de atenção, sob responsabilidade e coordenação imediata da gerência, de modo a manter um bom fluxo e contrafluxo com a RAS. No entanto, há evidências de que a baixa capacidade gerencial limita o acesso aos serviços de saúde, produz concentração de esforços em princípios administrativos tradicionais ou defasados e dificulta a sistematização de dados $(8,9)$ e o planejamento de ações, acarretando insatisfação do usuário, conflitos interpessoais e sobrecarga de trabalho (10-12).
O gerenciamento na APS é sumariamente tratado nas publicações da PNAB de 2006 (13) e de 2011 (3), que definem: "compete às Secretarias Municipais de Saúde e ao Distrito Federal organizar, executar e gerenciar os serviços e ações de atenção básica" (13, p. 12; 3, p. 32). A PNAB de 2017 (14) retoma a necessidade da gerência local, recomendando a existência de um profissional dedicado a esse fim e definindo algumas de suas atribuições. Diferentemente do proposto na reforma da Secretaria da Saúde ocorrida no estado de São Paulo nos anos $1970(15,16)$, de acordo com a qual a função de gerência era privativa de médicos com especialização em saúde pública, o gerente agora recomendado deve ter "preferencialmente" nível superior, não ser integrante das equipes vinculadas à UBS e possuir experiência na atenção básica (14).

Idealmente, o gerente de UBS teria atribuições de planejamento, coordenação, direção e controle das ações realizadas pela equipe e deveria cotejar conhecimentos e habilidades técnicas, administrativas e psicossociais, articular a equipe de trabalho e dar acesso aos usuários no planejamento participativo (6-9). Nessa perspectiva, o gerente é um agente-chave, sendo o responsável pela coordenação do trabalho da equipe e pela coerência das ações com os princípios e diretrizes da PNAB. No entanto, a literatura sobre o tema aponta a quase inexistência da gerência técnica na APS do SUS, seja pela ausência de um profissional que a exerça ou pela descaracterização desse papel (17-19). A gerência é hoje caracterizada como sendo absorvida por atividades mais administrativas, às quais se somam ações de assistência direta, com pouco tempo disponível para o trabalho de coordenação técnica da equipe $(8-10,18,20)$.

Por outro lado, a descentralização da gestão para os municípios e as políticas de incentivo para implementação da Estratégia Saúde da Família (ESF) como modelo organizacional prioritário ampliaram o número de serviços e redefiniram as bases nas quais se dá a gestão do trabalho na APS, estabelecendo uma nova relação entre gestão municipal e gerenciamento das $\operatorname{UBS}(9,21)$. Essas medidas permitiram avançar na implementação das diretrizes ético-normativas do SUS sinteticamente representadas nos princípios de universalidade, equidade e integralidade (3-5) e redefiniram o papel da gerência das UBS.

Nesse contexto complexo, o presente estudo objetiva descrever as características da gerência de unidades de APS em relação à coordenação do trabalho e à gestão municipal e discutir suas implicações para a efetivação de serviços de APS baseados nas diretrizes e pressupostos do SUS e coerentes com as proposições de Alma-Ata para produção do cuidado.

\section{MATERIAIS E MÉTODOS}

O presente estudo, descritivo, quantitativo e transversal, utilizou dados sobre gerência obtidos pela aplicação do Questionário de Avaliação da Qualidade de Serviços de Atenção Básica (QualiAB) (22) e pela ficha de cadastro dos gerentes das UBS. O QualiAB foi aplicado entre outubro e dezembro de 2014, a partir de adesão voluntária, em cinco regiões de saúde do estado de São Paulo. Essas cinco regiões de saúde reúnem 68 municípios, com 1624623 habitantes (23), e 303 UBS (24). Participaram do estudo 157 UBS, localizadas em 41 (63,1\%) municípios de diferentes tamanhos, com predomínio daqueles de pequeno porte (<25000 habitantes) (23).

O QualiAB é um instrumento estruturado, de autorresposta, preenchido via web, que objetiva avaliar a qualidade organizacional dos serviços de APS. O instrumento foi desenvolvido em 2007 (22, 25) e atualizado em 2014 e 2016 (www. abasica.fmb.unesp.br). Tem resultados analisados segundo diferentes recortes $(26,27)$. A versão de 2014 era composta por 126 questões de múltipla escolha que geravam indicadores de assistência e de gerência. Neste estudo, foram analisadas 31 questões sobre gerência, que incorporam tanto aspectos mais diretamente relacionados ao gerenciamento local como alguns mais dependentes da gestão municipal, considerando-se que a interface entre ambos incide sobre a qualidade da atenção realizada.

Os passos da participação na pesquisa foram: adesão voluntária do município pelo secretário municipal de saúde, cadastro da unidade pela equipe local, criação de usuário e senha para responder ao questionário com garantia de sigilo e recomendação para que as respostas fossem definidas em conjunto com a equipe. Todo o processo foi realizado on-line, com termo de consentimento livre 
e esclarecido tanto para os secretários municipais como para os gerentes locais. A pesquisa foi aprovada pelo Comitê de Ética em Pesquisa da Faculdade de Medicina de Botucatu - UNESP (parecer 1.314.674).

\section{RESULTADOS}

\section{Características gerais dos serviços}

Das 157 unidades participantes, 136 $(86,6 \%)$ eram diretamente vinculadas à gestão municipal e $17(10,8 \%)$ prestavam serviços por meio de contratos de gestão com Organizações Sociais/Fundações. Os seguintes arranjos organizacionais foram observados: 67 (42,7\%) unidades de saúde da família (USF), $58(36,9 \%)$ UBS "tradicionais" (equipes compostas por médicos de diferentes especialidades, sem agentes comunitários), 29 (18,5\%) UBS “tradicionais" com programa de agentes comunitários de saúde ou com a presença de equipes de saúde da família e três $(1,9 \%)$ UBS "tradicionais" ou USF integrada a pronto-atendimento.

O período de funcionamento das unidades era: em $114(72,6 \%)$, todas as manhãs e tardes; em $12(7,6 \%)$, todas as manhãs, tardes e noites; e 31 unidades $(19,4 \%)$ funcionavam em apenas um dos períodos. Quanto à localização geográfica, 95 (60,5\%) estavam localizadas em região urbana periférica, $49 \quad(31,2 \%)$ em região urbana central e $13(8,3 \%)$ em zona rural.

\section{Perfil dos gerentes ou responsáveis pela coordenação das unidades}

No momento de aplicação do questionário, oito $(5,0 \%)$ unidades não possuíam gerente e oito $(5,0 \%)$ eram gerenciadas por secretários municipais de saúde. $\mathrm{O}$ perfil dos gerentes locais está descrito na tabela 1. Como mostra a tabela, quase $80 \%$ dos gerentes eram enfermeiros e desempenhavam múltiplas ações, conforme descrito na tabela 2. De fato, nenhum dos enfermeiros que desenvolvia atividade de gerência declarou dedicação exclusiva a esse cargo.

\section{Interface entre a gestão municipal e o gerenciamento local}

$\mathrm{O}$ relacionamento entre a gerência das unidades e a gestão municipal se dava principalmente por meio de

TABELA 1. Características dos gerentes de 157 unidades básicas de saúde em 41 municípios do centro-oeste paulista, Brasil, 2014

\begin{tabular}{|c|c|c|}
\hline Característica & No. & $\%$ \\
\hline \multicolumn{3}{|l|}{ Sexo } \\
\hline Feminino & 141 & 89,8 \\
\hline Masculino & 16 & 10,2 \\
\hline \multicolumn{3}{|l|}{ Profissional que exerce a gerência da Unidade } \\
\hline Enfermeiro & 124 & 79,0 \\
\hline Outro & 17 & 10,8 \\
\hline Secretário municipal de saúde & 8 & 5,1 \\
\hline Assistente social & 6 & 3,9 \\
\hline Médico & 1 & 0,6 \\
\hline Unidade não possui gerente & 1 & 0,6 \\
\hline \multicolumn{3}{|l|}{ Formação dos gerentes que responderam "Outro" } \\
\hline Técnico de enfermagem & 6 & 35,2 \\
\hline Auxiliar de enfermagem & 4 & 23,5 \\
\hline Nutricionista & 2 & 11,8 \\
\hline Administrador & 1 & 5,9 \\
\hline Advogado & 1 & 5,9 \\
\hline Farmacêutico & 1 & 5,9 \\
\hline Contadora & 1 & 5,9 \\
\hline Auxiliar de consultório dentário & 1 & 5,9 \\
\hline \multicolumn{3}{|l|}{ Tempo de atuação na saúde (anos) } \\
\hline$<1$ & 7 & 4,4 \\
\hline 1 a 3 & 33 & 21,0 \\
\hline 4 a 7 & 47 & 30,0 \\
\hline 8 a 15 & 47 & 30,0 \\
\hline$>15$ & 23 & 14,6 \\
\hline \multicolumn{3}{|l|}{ Tempo de atuação na unidade atual (anos) } \\
\hline$<1$ & 55 & 35,0 \\
\hline 1 a 3 & 54 & 34,4 \\
\hline 4 a 7 & 26 & 16,6 \\
\hline 8 a 15 & 19 & 12,1 \\
\hline$>15$ anos & 3 & 1,9 \\
\hline \multicolumn{3}{|l|}{ Vínculo empregatício } \\
\hline Empregado público Consolidação das Leis do Trabalho (CLT) & 58 & 36,9 \\
\hline Servidor público estatutário & 40 & 25,5 \\
\hline Contrato CLT & 36 & 22,9 \\
\hline Cargo comissionado & 19 & 12,1 \\
\hline Contrato temporário pela administração pública regido por legislação especial & 2 & 1,3 \\
\hline Contrato temporário por prestação de serviço & 2 & 1,3 \\
\hline \multicolumn{3}{|l|}{ Carga horária semanal da gerência } \\
\hline 40 horas & 140 & 89,2 \\
\hline 30 horas & 13 & 8,3 \\
\hline 20 horas & 1 & 0,6 \\
\hline Outra & 3 & 1,9 \\
\hline
\end{tabular}

reuniões agendadas de acordo com o surgimento de problemas (68 unidades, $43,3 \%$ ) ou por meio de reuniões periódicas ou visitas para supervisão técnica programada (63 unidades, 40,1\%). Oito unidades $(4,9 \%)$ não possuíam mecanismos formais de articulação com o nível municipal. Dessas, duas eram gerenciadas pelo secretário municipal de saúde.

\section{Infraestrutura e insumos básicos}

Quanto a estrutura física e equipamentos, 107 unidades $(68,2 \%)$ relataram bom estado de conservação, 106 (67,5\%) relataram uma estrutura com ventilação e iluminação adequada, 149 (94,9\%) informaram possuir banheiros para usuários, $149(94,9 \%)$ tinham sala de espera, 115 $(73,2 \%)$ tinham número suficiente de 
TABELA 2. Atividades realizadas pelo enfermeiro que atuava como gerente em 124 unidades básicas de saúde em 40 municípios do centro-oeste paulista, Brasil, 2014ª

\begin{tabular}{lcc}
\hline Atividades & No. & $\%$ \\
\hline Recepção, avaliação e orientação de casos "extra" (demanda espontânea) & 98 & 79,0 \\
Registro de seus atendimentos em prontuário & 97 & 78,2 \\
Orientações para gestantes, hipertensos/diabéticos, entre outros & 95 & 76,6 \\
Orientação para uso correto de medicamentos & 95 & 76,6 \\
Consulta de enfermagem de casos "extra" & 94 & 75,8 \\
Supervisão do acolhimento realizado pelo auxiliar/técnico de enfermagem & 92 & 74,2 \\
Gerenciamento da unidade & 90 & 72,6 \\
Visita domiciliar & 88 & 71,0 \\
Notificação epidemiológica & 87 & 70,2 \\
Participação em reuniões de equipe multiprofissional & 87 & 70,2 \\
Aconselhamento em infecções sexualmente transmissíveis/Aids & 86 & 69,4 \\
Supervisão da equipe de enfermagem/agentes comunitários de saúde & 85 & 68,5 \\
Consulta de seguimento programático & 84 & 67,7 \\
Coleta de citologia oncótica & 81 & 65,3 \\
Coordenação das reuniões de equipe multiprofissional & 74 & 59,7 \\
Atividades de educação permanente da equipe & 74 & 59,7 \\
Grupos educativos/assistenciais & 73 & 58,9 \\
Atendimento de urgência/emergência & 72 & 58,1 \\
Avaliação dos faltosos em consulta & 66 & 53,2 \\
Prescrição de medicamentos para condições com protocolo pré-estabelecido & 37 & 29,8 \\
Participação no Conselho de Unidade de Saúde & 29 & 23,4 \\
Outros & 2 & 1,6 \\
\hline
\end{tabular}

a Resultados obtidos pela aplicação do instrumento QualiAB (www.abasica.fmb.unesp.br).

TABELA 3. Rede de apoio técnico e social para 157 UBS em 41 municípios do centrooeste paulista, Brasil, 2014

\begin{tabular}{lcc}
\hline Serviços de apoio municipais ou regionais & No. & $\%$ \\
\hline Centro de Referência de Assistência Social (CRAS) & 136 & 92,5 \\
Centro de Atenção Psicossocial (CAPS) & 101 & 68,7 \\
Ambulatórios de especialidades & 75 & 51,0 \\
Centros de Referência em Saúde do Trabalhador (CEREST) & 75 & 51,0 \\
Centro de Referência Especializado de Assistência Social (CREAS) & 69 & 46,9 \\
Ambulatório Médico de Especialidades & 62 & 42,2 \\
Serviços de atenção à mulher & 55 & 37,4 \\
Serviços de atenção ao idoso & 43 & 29,3 \\
Equipes multiprofissionais & 40 & 27,2 \\
Serviços de atenção à criança & 37 & 25,2 \\
Núcleo de Apoio à Saúde da Família (NASF) & 35 & 23,8 \\
Ações comunitárias ligadas a igrejas & 24 & 16,3 \\
Organizações não governamentais & 22 & 15,0 \\
Outros & 11 & 7,5 \\
Não tem acesso a serviços de apoio & 3 & 2,0 \\
\hline
\end{tabular}

${ }^{a}$ Resultados obtidos pela aplicação do instrumento QualiAB (www.abasica.fmb.unesp.br).

cadeiras, $110(70,1 \%)$ tinham banheiro no consultório ginecológico, 106 (67,5\%) tinham consultórios em número suficiente, $141(89,8 \%)$ tinham mesa para exame ginecológico, $123(78,3 \%)$ tinham geladeira exclusiva para vacinas, $67(42,7 \%)$ tinham carrinho de emergência e 50 (31,8\%) tinham desfibrilador.

Do total de serviços, 38 (24,2\%) não dispensavam medicamentos; 20 (12,7\%) referiram falta eventual dos medicamentos para hipertensão e diabetes, sendo que $27(16,6 \%)$ não dispensavam esses medicamentos; e $96(61,1 \%)$ possuíam todos os medicamentos legalmente previstos. Além disso, 151 (96,2\%) unidades distribuíam preservativo masculino, 127 $(80,9 \%)$ distribuíam anticoncepcional oral e duas $(1,3 \%)$ não dispensavam métodos contraceptivos.
Entre os serviços que ofereciam vacinação, a BCG era aplicada em 105 (66,9\%) e a pneumocócica 23 valente era aplicada em 91 (58,0\%). A vacinação não era realizada em 25 serviços (22,3\%).

Os exames realizados nas unidades eram: hemoglicoteste (HGT) em 154 $(98,1 \%)$, teste de gravidez na urina em $131(83,4 \%)$, teste rápido para HIV em 74 $(47,1 \%)$, teste rápido para sífilis em $72(45,9 \%)$, testes de hepatite B em 35 (22,3\%), hepatite C em 37 (23,6\%), eletrocardiograma (ECG) em oito $(51,0 \%)$ e coleta de exames clínicos laboratoriais em $102(65,0 \%)$ serviços.

\section{Acesso e composição da rede de atenção}

O tempo médio de espera entre o encaminhamento e a consulta para especialidades nos serviços de referência era de 1 a 3 meses, com maior espera nas seguintes especialidades: oftalmologia, otorrinolaringologia, ortopedia, gastrenterologia, cardiologia, neurologia, psiquiatria e fisioterapia. A tabela 3 mostra a rede de apoio municipal ou regional para os serviços de APS.

\section{Investimentos em formação continuada - matriciamento e oportunidades de formação}

O matriciamento, ou seja, supervisão técnica como forma de educação permanente, era feito em 26 (16,6\%) unidades pelo Núcleo de Apoio à Saúde da Família (NASF) e em 49 unidades (31,2\%) por equipe multiprofissional externa à unidade; 60 (38,2\%) unidades não contavam com nenhum tipo de matriciamento. $\mathrm{O}$ profissional que mais participou das estratégias de capacitação e formação continuada foi o enfermeiro, em 147 unidades $(93,6 \%)$, seguido pelo médico em 110 (70,1\%), pelo auxiliar/técnico de enfermagem em $97(61,8 \%)$ e pelo pessoal administrativo em 50 unidades $(31,8 \%)$. As temáticas mais abordadas nas formações foram: infecções sexualmente transmissíveis (IST) e Aids, em 113 (72,0\%), Saúde da Mulher em 99 (63,1\%) e acolhimento e atenção à demanda espontânea em $85(54,1 \%)$ ).

\section{Gerenciamento local e coordenação do trabalho}

Quanto à periodicidade das reuniões de equipe no ano anterior ao preenchimento 
do questionário, 60 unidades $(38,2 \%)$ referiram ter sido semanal e $39(24,8 \%)$ mensal. Informaram ausência de periodicidade ou de reuniões 23 unidades $(14,6 \%)$. As principais pautas das reuniões de equipe no último ano haviam sido: em 141 , rotinas da unidade $(89,8 \%)$, em $135(86,0 \%)$, organização do trabalho, informes em $128(81,5 \%)$, planejamento de ações em $127(80,9 \%)$, atualizações técnicas em $103(65,6 \%)$ e discussão de casos em $95(60,5 \%)$.

Os principais obstáculos citados para um bom desempenho das UBS, dificultando a coordenação do trabalho, estão listados na tabela 4 .

\section{Planejamento e avaliação}

A definição da área de abrangência das unidades era feita de modo centralizado pela Secretaria Municipal de Saúde (SMS) e com base em critérios administrativos em 76 unidades $(48,4 \%)$; por processo participativo, levando em conta a realidade local e facilidade de acesso em 47 (29,9\%). Não possuíam definição de área de abrangência 22 unidades $(14,0 \%)$. Os sistemas de informação utilizados e os dados com alguma forma de registro de rotina podem ser observados na tabela 5 .

Nos 3 anos anteriores ao preenchimento do questionário, 111 (70,7\%) serviços haviam realizado estudos sobre a realidade local por meio de dados dos programas (pré-natal, atenção à criança, atenção a hipertensos e diabéticos). Em 33 unidades $(21,0 \%)$, nenhum tipo de levantamento havia sido realizado.

A participação em um ou mais processos avaliativos foi referida por 130 (82,8\%) serviços, sendo: $76(48,4 \%)$ no Programa Nacional de Melhoria do Acesso e da Qualidade da Atenção Básica (PMAQ), 62 unidades $(39,5 \%)$ com avaliações organizadas pela SMS, 45 unidades $(28,7 \%)$ com avaliações pelo sistema QualiAB, 31 (19,7\%) com avaliações organizadas pela própria unidade e sete $(4,3 \%)$ com outras avaliações.

Os principais desdobramentos induzidos por processos de avaliação foram: planejamento e reprogramação das atividades com participação da equipe multiprofissional em 40 unidades $(25,5 \%)$ e definição de um plano anual de atividades das unidades em 38 (24,2\%). Não tiveram acesso aos resultados das avaliações 29 unidades (17,8\%). Quanto às modificações induzidas pelas avaliações,
TABELA 4. Principais obstáculos para a melhoria da qualidade da atenção à saúde referidos por 157 UBS localizadas em 41 municípios do centro-oeste paulista, Brasil, 2014

\begin{tabular}{lcc}
\hline Obstáculos & No. & $\%$ \\
\hline Falta de contrarreferência dos serviços especializados & 78 & 53,8 \\
Falta de recursos humanos & 72 & 49,7 \\
Excesso de demanda & 70 & 48,3 \\
Inadequação do espaço físico & 68 & 46,9 \\
Inadequação da postura dos usuários & 67 & 46,2 \\
Má remuneração dos profissionais & 65 & 44,8 \\
Falta de política de pessoal por parte da Secretaria/Prefeitura & 55 & 37,9 \\
Falta de retaguarda de serviços especializados para referência & 50 & 34,5 \\
Falta de mobilização da comunidade & 44 & 30,3 \\
Necessidade de informatizar o registro de dados & 43 & 29,7 \\
Falta de articulação/interação com serviços de urgência/emergência & 32 & 22,1 \\
Oscilação nas diretrizes políticas locais, devido a troca de prefeito/secretário & 30 & 20,7 \\
Não cumprimento do horário médico & 29 & 20,0 \\
Falta de capacitação da equipe técnica de enfermagem e/ou de saúde bucal & 27 & 18,6 \\
Falta de compromisso dos profissionais médicos & 21 & 14,5 \\
Falta de realização do trabalho em equipe & 20 & 13,8 \\
Falta de capacitação adequada da equipe de nível universitário & 16 & 11,0 \\
Falta de medicamentos & 14 & 9,7 \\
Falta de compromisso da equipe técnica de enfermagem e/ou de saúde bucal & 12 & 8,3 \\
Falta de compromisso dos profissionais de nível universitário & 8 & 5,5 \\
Outros & 5 & 3,4 \\
Não existem obstáculos importantes a serem vencidos & 13 & 8,3 \\
\hline
\end{tabular}

${ }^{a}$ Resultados obtidos pela aplicação do instrumento QualiAB (www.abasica.fmb.unesp.br).

TABELA 5. Sistemas de informação e dados registrados rotineiramente em 157 UBS em 41 municípios do centro-oeste paulista, Brasil, 2014a

\begin{tabular}{lcc}
\hline Sistemas de informação & No. & $\%$ \\
\hline Sistema Informatizado do Pré-Natal (SIS Pré-Natal) & 103 & 70,1 \\
Sistema de Informações de Agravos de Notificação (SINAN) & 96 & 65,3 \\
Sistema de Informação da Atenção Básica (SIAB) & 82 & 55,8 \\
Sistema de informação próprio do município & 71 & 48,6 \\
Sistema de Informação em Saúde para a Atenção Básica (e-SUS/SISAB) & 67 & 45,6 \\
Sistema Informatizado de Cadastro e Acompanhamento de Hipertensos e Diabéticos & 48 & 32,7 \\
(SIS HIPERDIA) & & \\
Outros sistemas de informação & 31 & 21,1 \\
Gerenciamento de Informações Locais & 5 & 3,4 \\
Não alimenta nenhum sistema de informação & 8 & 5,4 \\
\hline Dados com registro de rotina & & \\
\hline Número de procedimentos & 146 & 99,3 \\
Consultas médicas & 141 & 95,9 \\
Consultas de enfermagem & 136 & 92,5 \\
Número de pacientes atendidos & 133 & 90,5 \\
Número de coletas de Papanicolaou & 127 & 87,8 \\
Número de atendimentos "extra" (casos de demanda espontânea) & 121 & 82,3 \\
Número de visitas domiciliares & 121 & 82,3 \\
Consultas odontológicas & 120 & 81,6 \\
Consultas por faixa etária & 113 & 76,9 \\
Número de vacinas realizadas & 110 & 74,8 \\
Número de grupos realizados & 90 & 61,2 \\
Consultas por equipe multiprofissional & 59 & 40,1 \\
Faltas em atendimentos agendados & 59 & 40,1 \\
Primeiro atendimento no ano (por paciente) & 37 & 25,2 \\
Outros & 10 & 6,8 \\
Não existe registro de dados & 1 & 0,7 \\
\hline
\end{tabular}

a Resultados obtidos pela aplicação do instrumento QualiAB (www.abasica.fmb.unesp.br). 
82 unidades $(52,2 \%)$ referiram a realização de mudanças no gerenciamento e organização da assistência e 21 (13,4\%) não realizaram modificações.

Dos serviços pesquisados, 72 (45,9\%) não possuíam conselho local de saúde da unidade. Nas unidades com conselho local, os principais temas abordados nas reuniões no último ano foram: em $61(71,8 \%)$, problemas relativos ao atendimento; em $44(51,8 \%)$ planejamento de atividades educativas na comunidade e diagnóstico e priorização dos problemas do território; em 27 $(31,8 \%)$, conferência municipal de saúde. Em seis unidades $(7,0 \%)$ não houve reunião no último ano.

As reclamações dos usuários podiam ser feitas: diretamente para a gerência em 122 (77,7\%) unidades; por caixa ou livro de sugestão/reclamações em 86 (54,8\%); por sistema de ouvidoria da SMS em 85 (54,1\%); diretamente com o nível central em 50 (31,8\%); e no Conselho Municipal de Saúde em 43 (27,4\%). Somente três $(1,9 \%)$ unidades referiram não possuir canais de reclamação.

\section{Organização do fluxo assistencial}

O gerenciamento do fluxo assistencial priorizou questões relacionadas à organização de estratégias de agendamento, de atendimento à demanda espontânea e medidas que viabilizam ações de vigilância em saúde.

$\mathrm{O}$ agendamento de consulta nos serviços era feito: com hora marcada para cada paciente em 60 unidades $(38,2 \%)$; para todos os pacientes no início do turno em 57 unidades $(36,6 \%)$; e para grupo de pacientes por hora em 31 unidades $(19,7 \%)$. Em nove unidades $(5,7 \%)$ as consultas não eram agendadas, só atendiam demanda espontânea.

Não realizavam atendimento da demanda espontânea quatro (2,5\%) serviços. Nas 153 unidades que realizavam esse atendimento, o fluxo era organizado por triagem médica ou de enfermagem segundo critérios de gravidade e/ou evolução aguda em 72 (47,1\%); em 45 (29,4\%) a própria recepção orientava o fluxo e encaminhava ou não para atendimento; em 32 unidades (20,9\%) o encaminhamento era feito por auxiliar ou técnico de enfermagem, com supervisão, segundo critérios de risco e/ou vulnerabilidade; e era feito por médico ou enfermeiro de acordo com protocolo de estratificação de risco em três unidades $(2,0 \%)$.
Quanto a medidas de apoio à vigilância em saúde, a avaliação dos resultados dos exames era feita em 89 unidades $(56,7 \%)$ quando os exames chegavam no serviço; em 45 unidades (28,7\%) quando o paciente comparecia para atendimento; em 18 unidades $(11,5 \%)$ quando os resultados chegavam na unidade, mas apenas para os exames considerados prioritários (mamografia, exames do pré-natal, exames de urgência, entre outros); e em cinco unidades $(3,2 \%)$ no dia agendado para a consulta, mesmo que o paciente faltasse.

A convocação de faltosos, em apoio à vigilância em saúde, era feita para: gestantes em $122(77,7 \%)$ unidades; vacinação em 119 (75,8\%); resultados de exames alterados em 116 (73,9\%); tuberculose ou hanseníase em 111 (70,7\%); recém-nascido de risco em 97 (61,8\%); recém-nascido em 87 (55,4\%); e revisão pós-parto/puerpério e adulto crônico (hipertensão arterial e diabetes) com risco de complicação em 82 unidades (52,2\%). A convocação de faltosos não era realizada em nove $(5,7 \%)$ unidades.

\section{DISCUSSÃO}

Diversas proposições de Alma-Ata por exemplo, questões relativas a descentralização da gestão, planejamento, avaliação, financiamento e uso de tecnologias - mantêm-se como desafios para muitos sistemas nacionais de saúde (28). Em termos operacionais, a Declaração apontava a necessidade de definir a quem caberia a responsabilidade de coordenar o trabalho e resolver problemas administrativos, técnicos e sociais relacionados às ações desenvolvidas em cada serviço, assinalando a importância da preparação de administradores e planejadores em todos os níveis do sistema (1).

No presente artigo, observamos que a maioria dos gerentes nas UBS tinha formação em enfermagem. A predominância de gerentes com formação em enfermagem e do sexo feminino também foi relatada em outros estudos (8, 17-20, 29-31). Embora a enfermagem seja uma das poucas carreiras na área de saúde que contempla conteúdos de administração, diferentes estudos $(30,32)$ apontam que esses conteúdos não são suficientes para preparar os profissionais para a complexidade de gerenciar serviços de APS. Somada à falta de formação específica para essa função, a multiplicidade de tarefas assumidas pelo gerente enfermeiro compromete a efetividade do trabalho de gestão $(9,11,33)$.

A formação para gerenciamento da APS foi secundarizada nas últimas décadas. As deficiências mais apontadas, não só no Brasil como na América Latina e Central, recolocam a necessidade de ampliar a formação dos gerentes em temáticas relativas aos campos da administração, epidemiologia e metodologia operacional $(19,34)$.

Em relação às dificuldades para o gerenciamento local, os obstáculos apontados concentram-se em itens "externos" à governabilidade da própria unidade, tais como falta de recursos humanos, espaço físico e "inadequação" na postura dos usuários em seguir as normas e limites de serviços que dificultem o acesso. Questões mais internas à organização do trabalho foram referidas com menor frequência, como a dificuldade em realizar trabalho em equipe ou a falta de capacitação ou compromisso de diferentes profissionais (9).

Para tratar dos obstáculos à gestão, é essencial a integração entre gerência e gestão municipal, que não pode ficar restrita aos momentos em que surgem problemas. Deve basear-se no conhecimento e compromisso dos sujeitos envolvidos com a efetivação de ações de APS coerentes com as diretrizes de integralidade, universalidade e equidade. A manutenção da estrutura e insumos necessários, aliada à relativa autonomia da gerência na coordenação do trabalho da equipe, devem ser expressão de um projeto que promova a saúde como direito, como proposto pelo SUS (3, 5, $9,31)$. O gerenciamento das unidades precisa atender as especificidades de cada região sem abdicar desse projeto e sem que haja omissão do papel de coordenar o planejamento e a organização do processo de trabalho $(8,9)$. As situações observadas de gerência exercida diretamente pelo secretário municipal de saúde mostram-se inadequadas, já que esse é um cargo de indicação política, sem exigência de formação na área e que requer atuação macroinstitucional $(8,31)$.

Somente $29,9 \%$ dos serviços definiram a área de abrangência por meio de planejamento participativo. O processo de territorialização (3), com a participação dos profissionais e da comunidade, permite conhecer a realidade da área onde a 
unidade está inserida e planejar ações que atendam às necessidades de saúde da população, ampliando a capacidade de resposta do serviço. Na organização do fluxo, destaca-se o elevado número de serviços que mantinham o agendamento de todos os usuários no início de cada período de atendimento, reproduzindo uma prática tradicional que amplia o tempo de espera de cada usuário e prevê uma elevada proporção de faltas para encaixe da demanda espontânea, que tende a ser priorizada.

$\mathrm{O}$ acesso ao atendimento pela demanda espontânea deve ser garantido sem substituir, nem colocar em segundo plano, o seguimento periódico dos que vivem, por exemplo, com condições crônicas, ou estão em determinadas fases do ciclo de vida, como crianças e idosos (17). As UBS são a principal porta de entrada do SUS e cabe a elas acolher, fortalecer o vínculo e possibilitar um cuidado com longitudinalidade (2). A pergunta que se faz é como conseguir que a garantia de atendimento à livre Fundo das Nações Unidas para Infância (UNICEF). Conferência internacional sobre cuidados primários de saúde, Alma-Ata-URSS, 6-12 de setembro de 1978: relatório final. Brasília: OMSUNICEF; 1979. Disponível em: http:// apps.who.int/iris/bitstream/handle / 10665 /39228/9241800011_por. pdf;jsessionid=7556C7A7DB6CE5C150FE 9B6089C479C 1 ? sequence $=5$ Acessado em 2 de outubro de 2017.

2. Starfield B, Shi L, Macinko J. Contribution of primary care to health systems and health. Milbank Q. 2005;83(3):457-502.

3. Brasil, Ministério da Saúde, Secretaria de Atenção à Saúde. Departamento de Atenção Básica. Política Nacional de Atenção Básica. Brasília: Ministério da Saúde; 2012. Disponível em: http:/ / 189.28.128.100/dab/docs/ publicacoes/geral/pnab.pdf Acessado em 15 de agosto de 2017.

4. Mendes EV. As redes de atenção à saúde. 2a ed. Brasília: Organização PanAmericana da Saúde; 2011.

5. Brasil, Ministério da Saúde. Portaria 399/2006. Diário Oficial da União. 2006;22 fev. Disponível em: http://bvsms.saude.gov.br/bvs/saudelegis/gm/2006/ prt0399_22_02_2006.html Acessado em 11 de agosto de 2017.

6. Castanheira ERL. A gerência na prática programática. Em: Schraiber LB, Nemes MIB, Mendes-Gonçalves RB, organizadores. Saúde do adulto: programas e ações na unidade básica. 2a ed. São Paulo: Hucitec; 2000. Pp. 222-33. demanda seja uma medida de cuidado integral e não uma simplificação da assistência. A predominância do pronto atendimento tende a reproduzir uma atenção pontual e dirigida à queixa, substituindo medidas de seguimento longitudinal e comprometendo a integralidade do cuidado.

Como limitações do presente estudo, devem-se reconhecer os limites da participação por adesão voluntária e dos instrumentos de autorresposta, em função da variação do rigor e compromisso dos participantes, além da impossibilidade de generalização dos achados. Entretanto, o presente estudo recoloca em questão a importância da gestão do trabalho para efetivação de uma APS que siga as bases lançadas na Declaração de AlmaAta e reafirmadas internacionalmente e no Brasil.

No contexto atual, faz-se necessário (re)investir na formação de gestores como estratégia para o desenvolvimento de processos de trabalho coerentes com os princípios de uma APS integral e com

\section{REFERÊNCIAS}

7. Peduzzi M, Carvalho BG, Mandú ENT, Souza GC, Silva JAM. Trabalho em equipe na perspectiva da gerência de serviços de saúde: instrumentos para a construção da prática interprofissional. Physis. 2011;21(2):629-46.

8. Fernandes JC, Cordeiro BC. O gerenciamento de Unidades Básicas de Saúde no olhar dos enfermeiros gerentes. Rev Enferm UFPE on line. 2018;12(1):194-202.

9. Bazzo-Romagnolli AP, Gimenez-Carvalho B, Almeida-Nunes EFP. Gestão de unidade básica de saúde em municípios de pequeno porte: instrumentos utilizados, facilidades e dificuldades relacionadas. Rev Gerenc Polit Salud (Colombia). 2014;13(27):168-80.

10. Weirich CF, Munari DB, Mishima SM, Bezerra ALQ. O trabalho gerencial do enfermeiro na rede básica de saúde. Texto Contexto - Enferm. 2009;18(2):249-57.

11. Andre AM, Ciampone MHT, Santelle O. Tendência de gerenciamento de unidades de saúde e de pessoas. Rev Saude Publica. 2013;47(1):158-63.

12. Passos JP, Ciosak SI. A concepção dos enfermeiros no processo gerencial em unidade básica de saúde. Rev Esc Enferm USP. 2006;40(4):464-8.

13. Brasil, Ministério da Saúde, Secretaria de Atenção à Saúde. Departamento de Atenção Básica. Política Nacional de Atenção Básica. Brasília: Ministério da Saúde; 2006. Disponível em: http://bvsms.saude.gov.br/ bvs/publicacoes/politica_nacional_ atencao_basica_2006.pdf Acessado em 11 de agosto de 2016. capacidade de dar respostas às necessidades de saúde. Cabe à gerência local, com apoio técnico e político da gestão municipal, o papel de articular recursos e necessidades, em conjunto com a equipe e com a participação dos usuários, para efetivação de um trabalho capaz de promover a saúde como direito e condição de cidadania.

Agradecimentos. Este trabalho é parte do Projeto "Atenção Básica: atualização e validação do instrumento QualiAB para nível nacional", financiado pelo CNPq (Processo No 485848/2012-0) e um produto do mestrado de LON.

Conflitos de interesse. Nada declarado pelos autores.

Declaração. As opiniões expressas no manuscrito são de responsabilidade exclusiva dos autores e não refletem necessariamente a opinião ou política da RPSP/PAJPH ou da Organização PanAmericana da Saúde (OPAS).

14. Brasil, Ministério da Saúde. Portaria 2 436/2017. Diário Oficial da União. 2017;21 set. Disponível em: http:/ /bvsms. saude.gov.br/bvs/saudelegis/gm/2017/ prt2436_22_09_2017.html Acessado em 14 de outubro de 2016.

15. Marsiglia RMG. Comentário: curso de saúde pública em um semestre. Rev Saude Publica. 2006;40(5):778-9.

16. Mota A, Schraiber LB. Atenção primária no sistema de saúde: debates paulistas numa perspectiva histórica. Saude Soc. 2011;20(4):837-52.

17. Bloise MS. Os gerentes de unidades básicas de saúde do município do Rio de Janeiro: perfil, vivências e desafios [dissertação]. Rio de Janeiro: Escola Nacional de Saúde Pública Sergio Arouca, Fundação Oswaldo Cruz; 2015.

18. Ohira RHF, Cordoni Junior L, Nunes EFPA. Análise das práticas gerenciais na Atenção Primária à Saúde nos municípios de pequeno porte do norte do Paraná, Brasil. Cienc Saude Colet. 2014;19(11):4439-48.

19. Alves M, Penna CMM, Brito MJM. Perfil dos gerentes de unidades básicas de saúde. Rev Bras Enferm. 2004;57(4):441-6.

20. Falcão SMR, Sousa MNA. Gerenciamento da Atenção Primária à Saúde: estudos nas unidades básicas. Rev Enferm UFPE on line. 2011;5(6):1510-7.

21. Fertonani HP, de Pires DE, Biff D, Scherer MD. The health care model: concepts and challenges for primary health care in Brazil. Cienc Saude Colet. 2015;20(6):1869-78.

22. Castanheira ERL, Nemes MIB, Almeida MAS, Puttini RF, Soares ID, Patrício KP, et al. QualiAB: desenvolvimento e validação 
de uma metodologia de avaliação de serviços de atenção básica. Saude Soc. 2011;20(4):935-47.

23. Instituto Brasileiro de Geografia e Estatística (IBGE). Análise da disponibilidade domiciliar de alimentos e do estado nutricional no Brasil. Rio de Janeiro: IBGE; Cidades. Disponível em: http://www. cidades.ibge.gov.br/xtras/uf.php?lang= \&coduf $=35 \&$ search $=$ sao-paulo Acessado em 11 de agosto de 2016.

24. Cadastro Nacional de Estabelecimentos de Saúde (CNES). Brasília: CNES. Disponível em: http://www.cnes.datasus.gov. br Acessado em dezembro de 2014.

25. Castanheira ERL, Nemes MIB, Zarili TFT, Sanine PR, Corrente JE. Avaliação de serviços de atenção básica em municípios de pequeno e médio porte no estado de São Paulo: resultados da primeira aplicação do instrumento QualiAB. Saude Debate. 2014;38(103):679-91.

26. Sanine PR, Zarili TF, Nunes LO, Dias A, Castanheira ER. Do preconizado à prática: oito anos de desafios para a saúde da criança em serviços de atenção primária no interior de São Paulo, Brasil. Cad Saude Publica. 2018;34(6): e00094417.

27. Placideli N, Castanheira ERL. Atenção à saúde da pessoa idosa e ao envelhecimento em uma rede de serviços de atenção primária. Kairós Gerontol. 2017;20(2):247-69.

28. World Health Organization (WHO). The world health report 2008. Primary health care, now more than ever. Genebra: $\mathrm{WHO}$; 2008. Disponível em: http://www.who. int/whr/2008/en/ Acessado em 1 de dezembro de 2016

29. Carvalho BG, Peduzzi M, Nunes EFPA, Leite FS, Silva JAM. Gerência de unidade básica de saúde em municípios de diferentes portes: perfil e instrumentos gerenciais utilizados. Rev Esc Enferm USP. 2014;48(5):907-14.

30. Coatto A, Traldi MC. Competências gerenciais do enfermeiro na atenção primária. Saude Colet. 2011;8(52):180-6.

31. Nunes EFPA, Carvalho BG, Nicoletto SCS, Cordoni Junior L. Trabalho gerencial em
Unidades Básicas de Saúde de municípios de pequeno porte no Paraná, Brasil. Interface (Botucatu). 2016;20(58):573-84.

32. Aarestrup C, Tavares CMM. A formação do enfermeiro e a gestão do sistema de saúde. Rev Eletrônica de Enferm. 2008;10(1):228-34.

33. Feliciano KVO, Kovacs MH, Sarinho SW. Superposição de atribuições e autonomia técnica entre enfermeiras da estratégia saúde da família. Rev Saude Publica. 2010;44(3):520-7.

34. Motta PR. Manual sobre tendencias contemporáneas en la gestión de la salud. (Manual operativo $1.1 \mathrm{v}$ ). Washington: OPS; 1996.

Manuscrito recebido em 14 de janeiro de 2018. Aceito em versão revisada em 9 de agosto de 2018.
ABSTRACT

Importance of local management for delivery of primary health care according to Alma-Ata principles

Keywords
Objective. To describe the characteristics of the management process in primary health care units as well as the profile of managers, and to discuss the implications of these elements in implementing the principles of Brazil's Unified Health System principles in Brazil in accordance with the principles of the Alma-Ata Declaration. Method. This descriptive, cross-sectional study used data collected with the Primary Care Service Quality Assessment tool (QualiAB), a self-administered, web-based instrument. QualiAB was voluntarily answered by 157 unit managers from 41 municipalities in the state of São Paulo from October to December 2014.

Results. Of 157 units, $67(42.7 \%)$ were family health care units and 58 (36.9\%) were "traditional" units; $95(60.5 \%)$ were located in urban peripheries. At the time of the study, eight units $(5.0 \%)$ did not have a manager and eight $(5.0 \%)$ were managed by the city health secretary. Almost $80 \%$ of the managers were nurses and performed multiple tasks in addition to management. Multidisciplinary support (technical supervision as a means of continuing education) was available in 75 units (47.7\%); $60(38.2 \%)$ did not have any kind of multidisciplinary support. Participation in evaluative processes was mentioned in 130 units $(82.8 \%)$. The main results of evaluations were planning and reprogramming of activities with the engagement of the multiprofessional team in 40 units $(25.5 \%)$ and definition of an annual activity plan in $38(24.2 \%)$. Twenty-nine units (17.8\%) did not have access to the results of evaluations.

Conclusion. The study supports the importance of work process management and the need to (re)invest in training and upgrading of local management skills as a strategy to produce primary health care that is capable of promoting health as a right and a necessary condition of citizenship.

Health management; health services administration; primary health care; Brazil. 
RESUMEN Objetivo. Describir las características de la gestión de las unidades de atención primaria de salud y el perfil de los gerentes, y analizar las implicaciones de esos elementos en la puesta en práctica de los principios del Sistema Único de Salud de Brasil de forma coherente con las propuestas de Alma- Ata.

Importancia de la gestión local para una atención primaria de salud según las propuestas de Alma-Ata
Métodos. Estudio descriptivo, transversal, con datos recolectados a través del cuestionario de Evaluación de la Calidad de Servicios de Atención Básica (QualiAB), un instrumento autoadministrado a través de Internet. En total 157 gerentes de Unidades Básicas de Salud de 41 municipios del estado de São Paulo respondieron voluntariamente el QualiAB entre octubre y diciembre de 2014.

Resultados. De las 157 unidades, 67 (42,7\%) eran unidades salud de la familia y 58 (36,9\%) eran unidades básicas de salud de organización "tradicional"; 95 (60,5\%) estan ubicadas en una región urbana periférica. En el momento del estudio, ocho $(5,0 \%)$ unidades no poseían gerente y ocho $(5,0 \%)$ eran gestionadas por secretarios municipales de salud. Casi el 80\% de los gerentes eran enfermeros y desempeñaban múltiples funciones además de la gerencia. En 75 (47,7\%) unidades se disponía de apoyo multidisciplinario (supervisión técnica como forma de educación permanente); 60 (38,2\%) unidades no contaban con ningún tipo de apoyo multidisciplinario. La participación en procesos de evaluación fue referida por 130 (82,8\%) servicios. Las principales modificaciones inducidas por las evaluaciones fueron la planificación y reprogramación de las actividades con participación del equipo multiprofesional en 40 unidades $(25,5 \%)$ y la definición de un plan anual de actividades en 38 (24,2\%). No tuvieron acceso a los resultados de las evaluaciones 29 unidades $(17,8 \%)$.

Conclusión. El estudio subraya la importancia de la gestión del trabajo y la necesidad de reinvertir en la formación y valorización de la gestión local como estrategia para hacer efectiva una atención primaria de salud capaz de promover la salud como derecho y condición de ciudadanía.

Palabras clave Gestión en salud; administración de los servicios de salud; atención primaria de salud; Brasil. 